\title{
Correction to: Psychosocial and Clinical Correlates of Fatigue in Haemodialysis Patients: the Importance of Patients' Illness Cognitions and Behaviours
}

\author{
Joseph Chilcot ${ }^{1} \cdot$ Rona Moss-Morris $^{1} \cdot$ Micol Artom $^{1} \cdot$ Larissa Harden $^{1}$ • \\ Federica Picariello $^{1} \cdot$ Hector Hughes $^{2} \cdot$ Sarah Bates $^{1} \cdot$ Iain C. Macdougall $^{2}$
}

Published online: 20 October 2017

(C) International Society of Behavioral Medicine 2017

Correction to: International Journal of Behavioural Medicine, 23 (3), 271-281

https://doi.org/10.1007/s12529-015-9525-8

We wish to highlight a discrepancy with regard to the reported mean fatigue severity score (mean $=28.3$, standard deviation $=6.5$ ). Since publication we have followed this sample up over time and are currently evaluating the longitudinal trajectories of fatigue severity. We notice in our original paper, items on the Chalder Fatigue Scale (sometimes referred to as the Chalder Fatigue Questionnaire; CFQ) were scored using a 1-4 Likert scale [i.e. each item was scored; better than usual (1), no worse than usual (2), worse than usual (3) and much worse than usual (4)], instead of the commonly reported 0-3 scoring. In order to avoid discrepancies with any future published data and for consistency with the literature, we have recoded the CFQ using the recommended $0-3$ scoring giving a mean score of $17.3(\mathrm{~S} . \mathrm{D}=6.5)$. Since the $\mathrm{CFQ}$ has 11 -items, the corrected mean (derived from the $0-3$ scoring) is 11 points lower compared to the mean when using the 1-4 scoring. The atypical scoring used in our original paper does not impact upon the reported correlational analysis.

We also note a typo in Table 1. For the demographic and clinical characteristics reported at the bottom of the table, the means are in fact reported with standard errors and not standard deviations as originally stated. Standard deviation (S.D) can be calculated from the standards error using the following:

$\mathrm{S} . \mathrm{D}=\mathrm{SE}^{*}[\sqrt{N}]$

where SE is the standard error of the mean and $\mathrm{N}$ is the sample size.

The online version of the original article can be found at https://oi.org/ 10.1007/s12529-015-9525-8

Joseph Chilcot

joseph.chilcot@kcl.ac.uk

1 Health Psychology Section, Psychology Department, Institute of Psychiatry, Psychology and Neuroscience, King's College London, London, UK

2 Department of Renal Medicine, King's College Hospital, London, UK 